\title{
Left Hemisphere Lateralization for Language in Right-Handers Is Controlled in Part by Familial Sinistrality, Manual Preference Strength, and Head Size
}

\author{
Nathalie Tzourio-Mazoyer, ${ }^{1}$ Laurent Petit, ${ }^{1}$ Annick Razafimandimby, ${ }^{1,3}$ Fabrice Crivello, ${ }^{1}$ Laure Zago, ${ }^{1}$ Gael Jobard, ${ }^{1,2}$ \\ Marc Joliot, ${ }^{1}$ Emmanuel Mellet, ${ }^{1}$ and Bernard Mazoyer ${ }^{1,3,4}$ \\ ${ }^{1}$ Groupe d'Imagerie Neurofonctionnelle, Unité Mixte de Recherche 6232, Centre National de la Recherche Scientifique France, Commissariat à l'Energie \\ Atomique, France, ${ }^{2}$ Université de Caen, 14000 Caen, France, and ${ }^{3}$ Centre Hospitalier Universitaire de Caen, 14074 Caen Cedex, France, and ${ }^{4}$ Institut \\ Universitaire de France, 75000 Paris, France
}

We investigated the effects of familial sinistrality (FS+; presence of left-handedness in one's close relatives), manual preference strength (MPS), and head size on the hemispheric lateralization of language in right-handers. Functional magnetic resonance imaging was used to map 49 individuals while listening to a story in their mother tongue. We found that individuals who had both the FS + trait and weak MPS had no left hemisphere dominance for this lexicosyntactic task, whereas others showed a leftward functional asymmetry. In addition, the smaller the brain size, the smaller the leftward asymmetry for language, independent of FS and MPS. None of these effects were observed when the same subjects performed a spatial attention task that elicited right hemispheric functional asymmetry. These results demonstrate that the left hemisphere dominance for language in right-handers is a variable controlled, in part, by a number of specific factors, including FS, MPS, and head size.

\section{Introduction}

In $>90 \%$ of humans, both language and the preferred hand are hosted in the left hemisphere (LH), which has led to genetic theories linking hemispheric dominance with handedness and the emergence of language (Crow, 2010). Annett's (1998) theory, for example, postulates that a single dominant gene controls these two phenomena. In a recent study, we showed that the presence of left-handedness among a subject's first-order relatives (parents and siblings), a trait referred to as positive familial sinistrality $(\mathrm{FS}+)$, results in a $10 \%$ reduction of the left planum temporale (PT) surface area, a part of the auditory cortex involved in speech processing. This effect was independent of the subject's handedness and was not observed in the right PT (Tzourio-Mazoyer et al., 2010). This finding questions the classic model of congruent hand/language hemispheric dominance and suggests that the FS + trait is associated with reduced LH dominance for language. This hypothesis was first postulated by Hécaen et al. (1981), who observed patient recovery from aphasia and reported that FS + left-handers exhibit the lowest LH dominance for language. Using functional magnetic resonance imaging (fMRI), Hund-Georgiadis et al. (2001) later confirmed that normal FS + left-handers have reduced LH lateralization for language. However, our report (Tzourio-Mazoyer et al., 2010) indi-

\footnotetext{
Received May 21, 2010; revised July 31, 2010; accepted Aug. 6, 2010.

We are deeply indebted to Guy Perchey and Mathieu Vigneau for their help with data acquisition and analysis, and to Marie-Renée Turbelin for the acquisition of the spatial and language batteries.

Correspondence should be addressed to Dr. Nathalie Tzourio-Mazoyer, Groupe d'Imagerie Neurofonctionnelle, UMR6232, Commissariatà l'Energie Atomique, Centre National de la Recherche Scientifique, GIP Cyceron, Boulevard Henri Becquerel, BP 5229, F-14074 Caen Cedex, France. E-mail: tzourio@cyceron.fr.

D0I:10.1523/JNEUROSCI.2593-10.2010

Copyright $\odot 2010$ the authors $\quad$ 0270-6474/10/3013314-05\$15.00/0
}

cated that the presence of left-handers in the subject's family, rather than the subject's own handedness, is associated with a reduction in the left PT surface area, suggesting that language and hand hemispheric lateralization may be under the control of different influences, either genetic or epigenetic.

A way to further investigate this issue would be to assess whether the FS+ trait decreases language-related functional asymmetries in right-handers. Right-handedness is not a binary trait, as individuals describing themselves as right-handers exhibit a wide range of values for manual preference strength (MPS). Studies using functional transcranial Doppler ultrasonography have shown an increased occurrence of right hemisphere $(\mathrm{RH})$ dominance for language in right-handers lacking strong MPS (Knecht et al., 2000a). In addition, Crow et al. (1998) and Leask and Crow (2006) reported that MPS, rather than handedness per se, is related to language performance, with individuals with lower MPS having decreased language performance. According to these authors, the underlying phenomenon could be an association with weak MPS, whatever the preferred hand, with a reduction in the hemispheric functional asymmetry for language.

The aim of the present study was to investigate whether FS and/or MPS are associated with LH dominance for language and verbal abilities in right-handers. A hemispheric functional lateralization index (HFLI) was computed from blood oxygenation level-dependent (BOLD)-fMRI images acquired during a speech comprehension task in right-handers. Because we previously observed an influence of head size on language lateralization (Josse et al., 2006), the skull perimeter of each subject was measured. Finally, to evaluate the specificity of the effect of FS, MPS, and/or head size on language hemispheric asymmetries, visuospatial 
Table 1. MPS, story comprehension score, verbal and visuospatial global scores, and language and spatial attention HFLI (left-right) in the four FS and MPS subject subgroups

\begin{tabular}{|c|c|c|c|c|}
\hline & $\begin{array}{l}\text { FS-/MPS+ } \\
(22,8 \mathrm{~W})\end{array}$ & $\begin{array}{l}\text { FS-/MPS- } \\
(11,3 W)\end{array}$ & $\begin{array}{l}\text { FS+/MPS+ } \\
(9,4 \mathrm{~W})\end{array}$ & $\begin{array}{l}\text { FS+/MPS- } \\
(7,6 \mathrm{~W})\end{array}$ \\
\hline anguage HFLI & $22.0 \pm 35$ & $50.19 \pm 18$ & $33.7 \pm 25$ & $5.4 \pm 41$ \\
\hline Spatial attention HFLI & $-28.3 \pm$ & $-34.8=$ & $-32.4 \pm 13$ & $-27.0 \pm 14$ \\
\hline $\begin{array}{l}\text { Comprehension score } \\
\text { (maximum 11) }\end{array}$ & $9.23 \pm 1.1$ & $9.36 \pm 1.1$ & $9.37 \pm 0.8$ & $7.7 \pm 1.4$ \\
\hline Global verbal score & $0.038 \pm 0.67$ & $0.047 \pm 0.40$ & $0.091 \pm 0.63$ & $-0.31 \pm 0.66$ \\
\hline isuospatial score & $0.052 \pm 0.79$ & $0.23 \pm 0.69$ & $-0.14 \pm 0.40$ & $-0.34 \pm 0.92$ \\
\hline
\end{tabular}

Values are given as the mean $\pm S D$. Number of subjects and number of women $(W)$ are shown in parentheses.

skills and spatial attention HFLIs were also documented in these individuals.

\section{Materials and Methods}

Subjects. Forty-nine right-handed healthy volunteers (22 women) gave written informed consent to participate in the study, which was approved by our local ethics committee. The subjects' mean age \pm SD was $29 \pm 7$ years, their mean level of education was $16 \pm 3$ years of schooling since primary school. The subjects' mean skull perimeter, measured at the level of the eyebrows, passing at the top edge of the ears and along the occipital bump, was $57.0 \pm 2.0 \mathrm{~cm}$.

Based on the subjects' scores on the Edinburgh Inventory (90 \pm 15 ; range, 50-100), individuals were classified into two categories: strong manual preference (MPS + , Edinburgh score $=+100, N=31$ ) or absence of a strong manual preference (MPS,$- N=18$ ).

Thirty-three subjects had no history of familial sinistrality (FS-), whereas 16 were FS + . No significant association was found between the FS and MPS traits ( $p=0.53$, Fisher exact test).

Subjects' global verbal abilities were evaluated by a battery of six tests: recall a 15-word list, semantic verbal fluency, vocabulary extent, reading and listening span (Daneman and Carpenter, 1980), and rhyming. A global verbal score was calculated as the average of the standardized scores from the six tests.

Subjects' visuospatial abilities were assessed with the mental rotation test, which evaluates the ability for rotation and spatial manipulation of mental images; the "Corsi Block Test," which evaluates visuospatial short-term memory abilities; and a home-made 3D maze test to evaluate topographic orientation skills. A global visuospatial score was calculated as the average of the standardized scores from the three tests.

Age, educational level, skull perimeter, verbal score, and visuospatial scores did not significantly differ among the four subgroups of subjects defined by the FS and MPS traits (Table 1). Skull perimeter, rather than total intracranial volume (TIV), was included to account for head size effects, because MRI data were of insufficient quality to derive reliable TIV values in some subjects.

$f M R I$ tasks. To evaluate the hemispheric lateralization of language, we used a story-listening task that alternated 30 s blocks of a narrative spoken in the subjects' mother tongue (French) with 30 s blocks of a narrative in an unknown language (Tamil). This paradigm has been shown to elicit reproducible leftward hemispheric asymmetries of lexicosyntactic areas (Mazoyer et al., 1993; Razafimandimby et al., 2007). Immediately after completing fMRI acquisition, the subjects answered an 11 item questionnaire designed to evaluate their comprehension and recall of the content of the story they just heard.

To evaluate hemispheric functional lateralization of spatial attention, the same subjects performed $30 \mathrm{~s}$ blocks of a line bisection judgment (LBJ) task that alternated with blocks for a saccadic eye movement task designed to match the eye movements and motor response components of the LBJ task. During LBJ, horizontal segments bisected with a vertical tick mark were visually presented every $2 \mathrm{~s}$ on a screen. Subjects had to decide whether the vertical tick mark bisected the horizontal segment in its exact center or slightly to the left or right of its center and respond by pressing a three-button response pad. Segments subtended a $6-10^{\circ}$ visual angle and were randomly positioned on the screen. Over the four LBJ blocks, 60 items were presented with an equal number of center, left, and right bisections.

Image acquisition. MRI was performed using a Philips Achieva 3tesla scanner. Whole brain functional volumes were acquired with a BOLDfMRI $\mathrm{T}_{2}{ }^{*}$-weighted echo-planar sequence $(240$ volumes; repetition time $=2 \mathrm{~s}$; echo time $=35 \mathrm{~ms}$; flip angle $=80^{\circ} ; 31$ axial slices; $3.75 \mathrm{~mm}^{3}$ isotropic voxel size). The first four volumes of each sequence were discarded to allow for stabilization of the MR signal. Before functional acquisition, high-resolution, three-dimensional (3D), T1-weighted volume and $\mathrm{T} 2{ }^{\star}$-weighted multislice (T2* MS) images were acquired.

Image analysis. Preprocessing was based on Statistical Parametric Mapping subroutines (SPM5, Wellcome Department of Neurology; http://www.fil.ion.ucl.ac.uk/spm). Anatomical T1-weighted volumes were spatially normalized by aligning individual anatomical volumes to specific cerebral tissue templates built from the T1-weighted images of 80 right-handed subjects (40 men) acquired with the same scanner and acquisition parameters. Spatial normalization parameters were set to their SPM5 default values, providing a 3D, spatially normalized deformation field for each subject.

Each functional run was corrected for slice timing and motion, and registered onto the $\mathrm{T} 2^{*}$-MS volume. Combining the $\mathrm{T} 2^{*}$-MS to T1weighted registration parameters and the spatial normalization parameters, functional images were resampled into the template space and spatially smoothed (Gaussian $6 \mathrm{~mm}$ full width at half-maximum filter). For each subject, the effects of interest were modeled by boxcar functions computed with paradigm timing and convolved with a standard hemodynamic response function (SPM5). Finally, the effect of interestsrelated contrast maps (French - Tamil and LBJ - saccades) were calculated.

The functional lateralization for language and spatial attention was assessed using Wilke and Lidzba's (2007) method, which provides threshold-free and robust estimates of the HFLI. The index was computed using the 5000 most activated voxels, discarding clusters with $<50$ voxels. For both tasks, the inclusive mask was the anatomical template excluding both the cerebellum and a left sensorimotor cortex cluster corresponding to the right-hand motor response assessed during the spatial attention task [3650 voxels $(-38,-20,64])]$.

Statistical analysis. To test whether FS and/or MPS were related in the same manner to verbal and/or visuospatial abilities, we ran a mixedmodel repeated-measures ANCOVA of the global verbal and visuospatial scores with "cognitive ability" as a two-level, within-subject factor (namely, language and visuospatial), and FS, MPS, and their interaction as between-subject factors. Gender, age, cultural level, and skull perimeter were included as covariates of interest.

To test whether FS and MPS were associated with the score obtained on the story questionnaire, we ran an ANCOVA of this score with FS, MPS, and their interaction as between-subject factors. Gender, age, skull perimeter, global verbal score, and level of education were included in the ANCOVA as covariates of interest.

Finally, to evaluate whether FS, MPS, and skull perimeter were related to hemispheric functional asymmetries for language, and to determine whether such relationships would be identical for the LBJ task, the HFLIs derived from the language and spatial attention tasks were entered in a mixed-model repeated-measures ANCOVA with cognitive task as a twolevel within-subject factor; FS, MPS, and their interaction as betweensubject factors; and skull perimeter and the comprehension score as covariates of interest. Gender and age were also included as additional confounding factors in the ANCOVA. Statistical analyses were performed using JMP software (version 7.0, SAS).

\section{Results}

\section{Relationship of FS and MPS with verbal and visuospatial abilities}

We found no effect of FS, MPS, their interaction, age, sex, or skull perimeter on the subjects' global verbal and visuospatial scores. The subjects' level of education was found to significantly and positively correlate with both scores $(p<0.0001)$. No interaction was found between any factor and the type of cognitive ability. 


\section{Relationship of FS and MPS with the comprehension score}

The average story comprehension score was $9.0 \pm 1.1$ (maximum $=11$ ), indicating that the subjects paid sustained attention to the story. ANCOVA revealed an interaction between FS and MPS $\left(F_{(40,1)}\right.$ $=4.38, p=0.042)$ together with a significant main effect of FS $\left(F_{(40,1)}=7.20, p=\right.$ $0.010)$. Post hoc Student's $t$ tests demonstrated that these findings were due to FS+/MPS - individuals having significantly lower scores than individuals of the three other groups (FS+/MPS - vs FS+/ MPS $+: t=2.65, p=0.01 ; \mathrm{FS}+/ \mathrm{MPS}-\mathrm{vs}$ $\mathrm{FS}-/ \mathrm{MPS}+: t=2.86, p=0.007$; FS + / MPS - vs FS-/MPS-: $t=2.68, p=$ 0.010 ) (for group average score values, see Table 1), whereas there was no difference among the three other groups $(p=0.97$, 0.75 , and 0.75 , respectively, for the comparison between FS+/MPS+ and FS-/ $\mathrm{MPS}+, \mathrm{FS}+/ \mathrm{MPS}+$ and FS-/MPS-, and FS - /MPS - and FS - /MPS + ). In addition, the story comprehension score was found positively correlate with the global verbal score $(r=0.75, p=0.0023)$.

\section{Neuroanatomofunctional pattern of each task}

Figure 1 shows the network of brain areas recruited by each task revealed by the sample probability map of the 5000 most activated voxels used for the computation of individual HFLIs.

During the speech-listening task, the most activated voxels were located bilaterally along the superior temporal sulcus from the temporal pole to the angular gyrus, in the inferior frontal gyri, and in the left precentral gyrus (Fig. 1). Leftward asymmetry was visible in the posterior part of the superior temporal sulcus, including the angular gyrus, in the upper part of the inferior frontal gyrus, and at the level of the precentral gyrus. The anterior part of the insula and the medial and orbital part of the superior frontal gyrus were activated, as well as the inferior part of the precuneus and calcarine cortex.

During the spatial attention task, the most activated voxels spilled bilaterally over the entire occipital cortex, extending to the intraparietal sulci with rightward asymmetry in the middle and inferior occipital gyri (Fig. 1). In addition to the most anterior part of the insular lobes, activated voxels ran along the precentral sulci in the frontal lobe toward the inferior frontal sulcus and the opercular part of the inferior frontal gyri with rightward asymmetry. In the medial wall, the presupplementary motor area was included, as well as the posterior part of the superior frontal gyrus.

\section{HFLI distribution}

The sample distributions of HFLI values for the language and spatial attention tasks are shown in Figure 1, and the subgroup average values are given in Table 1 . Average HFLIs for language were positive (mean $\pm \mathrm{SD}, 28.0 \pm 33.8$; range, $-71-67$ ), indicating a leftward hemispheric asymmetry, whereas average spatial attention HFLI values were negative $(-30.3 \pm 22.4$; range $-74-20$ ), indicating rightward hemispheric asymmetry.

The distribution of language HFLI values was skewed to the left and showed a larger variance than the distribution of spatial attention HFLI values, with the latter following a Gaussian distribution.

\section{HFLI ANCOVA}

The ANCOVA of HFLI values revealed a significant interaction among task, FS, and MPS ( $p=0.0030)$, a task by FS interaction $(p=0.0061)$, and a task by skull perimeter interaction $(p=$ $0.0052)$. A significant main effect of gender was present, with women being more leftward lateralized than men in both tasks; this effect being unraveled by the skull perimeter variable integration in the model (women vs men, $p=0.009$ ), the task $\times$ gender interaction was not significant ( $p=0.13$ ). We found no effect of the comprehension score nor interaction between score and task on HFLI values.

The task $\times$ FS $\times$ MPS interaction (Fig. 2) was due to the fact that, among FS + individuals, those with strong manual preference had larger HFLI values for the language task than those with weaker MPS (estimated HFLI mean \pm SD for FS $+/ \mathrm{MPS}+$, $40.0 \pm 10.0$; for FS+/MPS,$--3.6 \pm 12.0 ; p<0.05$, post hoc Student's $t$ test). In FS - subjects, the reverse situation was observed; subjects devoid of a strong manual preference had larger positive HFLI values for language (FS - /MPS - , $54.4 \pm 9.8$; FS - / MPS,$+ 25.8 \pm 6.6 ; p<0.05$, post hoc Student's $t$ test). Meanwhile, HFLI values for the spatial attention task were not influenced by FS, MPS, or their interaction, which also explains the task by FS interaction (Fig. 2).

These findings were found to be robust with respect to a potential bias due to the skewed nature of the language HFLI distribution because a nonparametric analysis performed after transforming HFLI values into ordinal variables led to identical conclusions.

The interaction between task and skull perimeter (Fig. 2) was due to a positive correlation between skull perimeter and the HFLI for language $(p=0.0046)$, but not the spatial attention HFLI $(p=0.58)$. 
HFLI speech comprehension
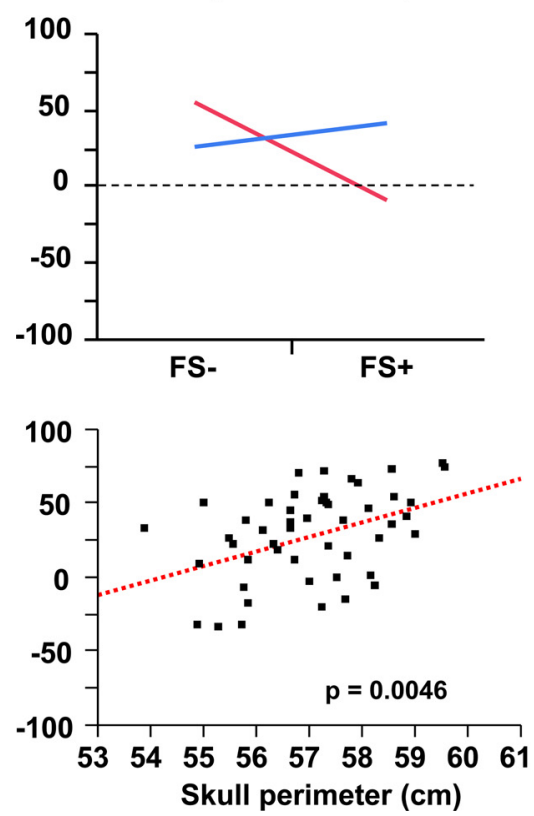

HFLI spatial attention
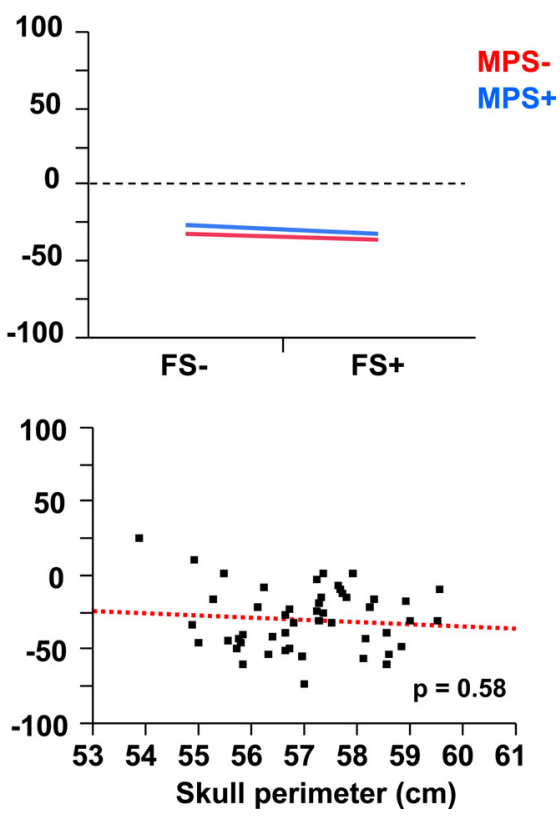

Figure 2. Effects of FS, MPS, and head size on HFLI values for language and spatial attention. Top left, MPS by FS interaction on the language HFLI. The plot shows the absence of leftward asymmetry in the FS +/MPS - group. Top right, MPS by FS interaction on the spatial attention HFLI. The plot shows no effect of these factors. Bottom left, Language HFLI values as a function of head size. The plot illustrates their positive correlation. Bottom right, Spatial attention HFLI values as a function of head size. The plot shows the absence of a similar relationship.

\section{Discussion}

The present results constitute the first evidence that two factors, namely the presence of left-handers among one's close relatives and the absence of a strong MPS, interact and result in reduced LH dominance for language in right-handers without any effect on their RH dominance for visuospatial attention. The interaction of these two factors also results in lower comprehension scores, but they have no significant impact on subjects' global verbal and visuospatial abilities. Thus, our findings identify FS and MPS as significant determinants of brain organization for language in right-handers.

The HFLI distribution shows that, in our group of righthanded subjects, the hemispheric asymmetries for language were more variable than those for visuospatial attention. The distribution we observed is consistent with that reported by Knecht et al. (2000a) when using functional transcranial Doppler ultrasonography (fCTD) in right-handers; a leftward tail corresponded to subjects without a leftward asymmetry. The observation of variability in language dominance in right-handers has also been documented in studies of crossed aphasia (Sweet et al., 1984; Coppens et al., 2002) and is consistent with reports of RH activation during functional imaging of language tasks in right-handers (Fischer et al., 1991; Crivello et al., 1995; Bakar et al., 1996; Tzourio et al., 1998). The results of the present study show that this variability is partly explained by the FS trait, in agreement with the hypothesis of Hécaen et al. (1981) and Hardyck and Petrinovich (1977) that FS + individuals have lower LH dominance for language. However, when this hypothesis was formulated, the authors mainly relied on the observation of FS + left-handers. Reduced LH language dominance in FS+ left-handers was also reported by other fMRI studies (Hund-Georgiadis et al., 2001; Szaflarski et al., 2002) and interpreted within the frame of common inheritance for hand and language dominance. A trend toward a higher occurrence of rightward lateralization in FS+ individuals was also described with FCTD in a population mixing left-handers and right-handers (Knecht et al., 2000b). Here, the observation of reduced LH functional dominance for language in FS+ right-handers, together with our recent report that FS+ individuals have less left planum temporale surface area independent of handedness (Tzourio-Mazoyer et al., 2010), strongly suggest that the heritability of hand and language lateralization is, at least in part, governed by different mechanisms.

Considering that the presence of FS and weak manual preference resulted in an absence of hemispheric asymmetry for language, one could consider that these factors may act on the same target. However, in the FS - subgroup MPS - subjects had larger HFLI values than MPS+ subjects. In addition, in the MPS + subgroup FS+ subjects had larger HFLI values than FS - subjects. Together, our results show that FS and MPS do not have simple additive effects; thus, they are likely to act through different mechanisms to set up the hemispheric specialization of language. Note that previous studies on language lateralization either compared groups of left-handers and right-handers (Tzourio et al., 1998; Cuzzocreo et al., 2009) or investigated the effects of MPS or FS in left-handers only (Szaflarski et al., 2002). The fact that the proportion of FS+/MPS - right-handed subjects in the general population is low $(\sim 10 \%)$ likely explains the absence of previous reports on the differential effect of FS and MPS on hemispheric dominance in language.

Comprehension scores and brain lateralization for language appeared to not be linearly related, probably because each variable is under the influence of different factors. Though educational level had a strong relationship with both the global verbal score and the comprehension score, it seems to not be related to the HFLI. In contrast, the language HFLI was linearly related to head size, a factor that does not seem to affect verbal abilities. Of course, one cannot exclude the possibility that lower scores during text recall may be related to subtle differences in asymmetries that could not be detected due to a lack of sensitivity. Nevertheless, FS interacting with MPS explains part of the variability in both comprehension scores and the hemispheric lateralization of speech, suggesting that both factors are involved in setting up hemispheric specialization and language abilities.

FS+/MPS - subjects had significantly lower comprehension scores than the other subjects, which supports Crow's hypothesis of the impact of MPS on verbal abilities (Crow et al., 1998; Leask and Crow, 2006). The fact that this effect was detected for comprehension scores rather than global verbal scores is likely to be related to insufficient statistical power of the present experimental design ( 0.53 for a 0.05 type I error) given the larger variability of global verbal score in these subjects. Further investigation on larger samples of individuals are thus needed for demonstrating that Crow's hypothesis holds for global verbal abilities.

The increased hemispheric dominance for language with head size, demonstrated here with fMRI, replicates our previous report 
on functional asymmetries of speech comprehension measured with PET (Josse et al., 2006). It reinforces the hypothesis of Ringo et al. (1994) that an increase in interhemispheric transfer time in large brains induces the clustering of functions that require fast processing in a single hemisphere. When listening to speech, speed is needed to process speech sounds; thus, the hypothesis of Ringo et al. (1994) appears to be useful for explaining the differential influence of brain volume. However, our present results concern leftward functional asymmetries in lexicosyntactic areas because the contrast between listening to a story in one's mother tongue to listening in an unknown foreign language eliminates the activation of primary auditory areas. In contrast to phonological processing, lexical and syntactic processing do not require fast processing, and the linear relationship between their leftward asymmetry and increase in head size is likely related to the development of preferential intrahemispheric connections between phonological and lexicosyntactic areas. The fact that such a relationship was not present for spatial attention asymmetries indicates that the hypothesis of Ringo et al. (1994) is particularly relevant for language functions because visuospatial attention does not call for fast processing.

Concerning the hemispheric lateralization of spatial attention, observations of left-handed patients with brain lesions have shown less RH dominance for attention in FS + left-handers (Hécaen et al., 1981). In the present study, we did not observe less rightward lateralization during spatial attention in FS+ righthanders, a relationship with MPS, or an interaction between these factors. Moreover, head size appears to have no relationship with the degree of hemispheric asymmetry for spatial attention tasks. Altogether, the findings show that, in right-handers, FS, MPS, and brain volume target language hemispheric organization rather than general hemispheric organization of the brain.

In summary, FS and MPS appear to interact in hemispheric lateralization for language and verbal memory in right-handers, but not in hemispheric lateralization for spatial attention in right-handers. Whether and how FS and MPS interact with handedness and the language production networks need further investigation to progress in our understanding of how hemispheric specialization is set up in humans. The association between left hemisphere specialization for language and FS + has also to be investigated in schizophrenic right-handed patients in which a lesser left hemisphere lateralization for language has been evidenced in the same task (Dollfus et al., 2005).

\section{References}

Annett M (1998) Handedness and cerebral dominance: the right shift theory. J Neuropsychiatry Clin Neurosci 10:459-469.

Bakar M, Kirshner HS, Wertz RT (1996) Crossed aphasia. Functional brain imaging with PET or SPECT. Arch Neurol 53:1026-1032.

Coppens P, Hungerford S, Yamaguchi S, Yamadori A (2002) Crossed aphasia: an analysis of the symptoms, their frequency, and a comparison with left-hemisphere aphasia symptomatology. Brain Lang 83:425-463.

Crivello F, Tzourio N, Poline JB, Woods RP, Mazziotta JC, Mazoyer B (1995) Intersubject variability in functional anatomy of silent verb generation: assessment by a new activation detection algorithm based on amplitude and size information. Neuroimage 2:253-263.

Crow TJ (2010) A theory of the origin of cerebral asymmetry: epigenetic variation superimposed on a fixed right-shift. Laterality 15:289-303.

Crow TJ, Crow LR, Done DJ, Leask S (1998) Relative hand skill predicts academic ability: global deficits at the point of hemispheric indecision. Neuropsychologia 36:1275-1282.

Cuzzocreo JL, Yassa MA, Verduzco G, Honeycutt NA, Scott DJ, Bassett SS (2009) Effect of handedness on fMRI activation in the medial temporal lobe during an auditory verbal memory task. Hum Brain Mapp 30:1271-1278.

Daneman M, Carpenter PA (1980) individual differences in working memory and reading. J Verb Learn Verb Behav 19:450-466.

Dollfus S, Razafimandimby A, Delamillieure P, Brazo P, Joliot M, Mazoyer B, Tzourio-Mazoyer N (2005) Atypical hemispheric specialization for language in right-handed schizophrenia patients. Biol Psychiatry 57:1020-1028.

Fischer RS, Alexander MP, Gabriel C, Gould E, Milione J (1991) Reversed lateralization of cognitive functions in right-handers. Brain 114:245-261.

Hardyck C, Petrinovich LF (1977) Left-handedness. Psychol Bull 84:385-404.

Hécaen H, De Agostini M, Monzon-Montes A (1981) Cerebral organization in left-handers. Brain Lang 12:261-284.

Hund-Georgiadis M, Lex U, von Cramon DY (2001) Language dominance assessment by means of fMRI: contributions from task design, performance, and stimulus modality. J Magn Reson Imaging 13:668-675.

Josse G, Hervé PY, Crivello F, Mazoyer B, Tzourio-Mazoyer N (2006) Hemispheric specialization for language: brain volume matters. Brain Res 1068:184-193.

Knecht S, Deppe M, Dräger B, Bobe L, Lohmann H, Ringelstein E, Henningsen H (2000a) Language lateralization in healthy right-handers. Brain 123:74-81.

Knecht S, Dräger B, Deppe M, Bobe L, Lohmann H, Flöel A, Ringelstein EB, Henningsen $\mathrm{H}$ (2000b) Handedness and hemispheric language dominance in healthy humans. Brain 123:2512-2518.

Leask SJ, Crow TJ (2006) A single optimum degree of hemispheric specialisation in two tasks, in two UK national birth cohorts. Brain Cogn 62:221-227.

Mazoyer B, Dehaene S, Tzourio N, Frak V, Cohen L, Murayama N, Levrier O, Salamon G, Mehler J (1993) The cortical representation of speech. J Cogn Neurosci 5:467-479.

Razafimandimby A, Maïza O, Hervé PY, Lecardeur L, Delamillieure P, Brazo P, Mazoyer B, Tzourio-Mazoyer N, Dollfus S (2007) Stability of functional language lateralization over time in schizophrenia patients. Schizophr Res 94:197-206.

Ringo JL, Doty RW, Demeter S, Simard PY (1994) Time is of the essence: a conjecture that hemispheric specialization arises from interhemispheric conduction delay. Cereb Cortex 4:331-343.

Sweet EW, Panis W, Levine DN (1984) Crossed Wernicke's aphasia. Neurology 34:475-479.

Szaflarski JP, Binder JR, Possing ET, McKiernan KA, Ward BD, Hammeke TA (2002) Language lateralization in left-handed and ambidextrous people: fMRI data. Neurology 59:238-244.

Tzourio N, Crivello F, Mellet E, Nkanga-Ngila B, Mazoyer B (1998) Functional dominance for speech comprehension in left handers versus right handers. Neuroimage 8:1-16.

Tzourio-Mazoyer N, Simon G, Crivello F, Jobard G, Zago L, Perchey G, Hervé PY, Joliot M, Petit L, Mellet E, Mazoyer B (2010) Effect of familial sinistrality on planum temporale surface and brain tissue asymmetries. Cereb Cortex 20:1476-1485.

Wilke M, Lidzba K (2007) LI-tool: a new toolbox to assess lateralization in functional MR-data. J Neurosci Methods 163:128-136. 